\title{
Erratum to "Genome engineering via homologous recombination in mouse embryonic stem (ES) cells: an amazingly versatile tool for the study of mammalian biology" [An Acad Bras Cienc 73(2001): 365-383] \\ CHARLES BABINET and MICHEL COHEN-TANNOUDJI
}

Unité de Biologie du Développement, CNRS URA 1960, Institut Pasteur, 75015 Paris, France

The Editor and the Publisher regret that the heading of Table IV in this review article was erroneously printed as "Some examples of knock-in" rather than "Some examples of conditional mutagenesis". They also apologize for errors in Figures 2, 3 and 5 whose corrected versions are reproduced in the following pages.

\section{CORRECTIONS IN THE TEXT}

- Page 369, column 2, lines 14 to 18 .

Reads:

"... Such an interference has been demonstrated in several studies (Fiering et al. 1999) and, in some cases can lead to hypomorph mutations by the introduction the selection sequences in the 5 , region of a gene."

Should read:

“... Such an interference has been demonstrated in several studies (Fiering et al. 1999) and, in some cases, can lead to hypomorph mutations by the introduction of the selection sequence in the 5' region of a gene."

- Page 371, column 1, lines 6 to 9 .

Reads:

"At the end of this scenario, the muted allele retains a $\operatorname{lox} P$ site; however, no indication of an interference of this site with genetic expression has ever been demonstrated."

E-mail: chbabi@pasteur.fr/m_cohen@pasteur.fr 
Should read:

"At the end of this scenario, the mutated allele retains a lox $P$ site; however, no indication of an interference of this site with genetic expression has ever been demonstrated."

- Page 378, column 1, lines 1-3.

Reads:

"introduction in ES cells of gene mutations eventually implied in a given differentiation pathway and should bring new light..."

Should read:

"introduction in ES cells of mutations into genes eventually implicated in a given differentiation pathway should bring new light..."

a)

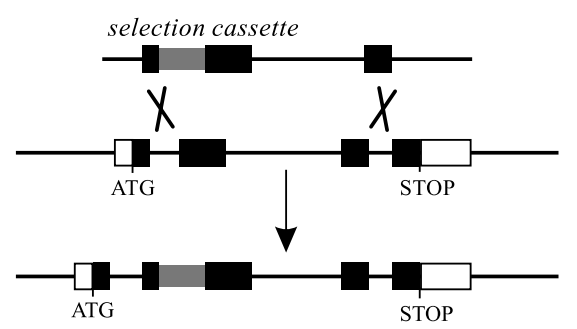

Knock-out: gene disruption by selection cassette insertion b) gene of interest (reporter gene, immortalizing gene, paralogue, mutated $c D N A, \ldots)$ selection

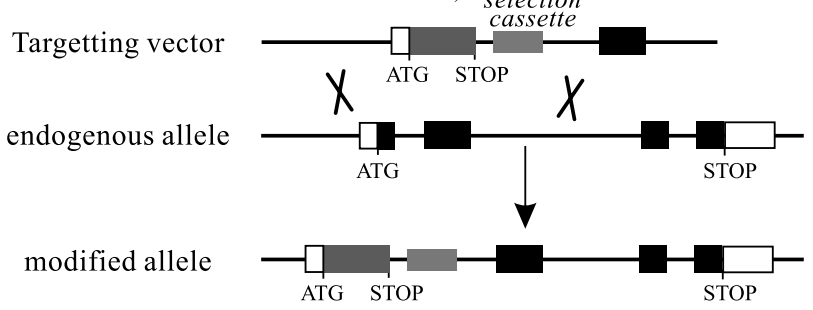

Knock-in: gene of interest expressed in place of the targetted gene

Fig. 2 - General principle of homologous recombination. a) "Knock-out". The targeting vector includes a selection cassette inserted in an exon (black rectangle: coding region; white rectangles: non coding regions) and surrounded by regions of homology with the target gene. Recombination with the endogenous gene occurs within these homologous sequences and results in the creation of a null allele in which disruption of the gene is induced by insertion of the selection cassette. b) "Knock-in". In this variant, besides invalidation of the target gene, a gene of interest is introduced in the locus. Following homologous recombination, the gene of interest is placed under the control of the promoter and regulatory sequences of the target gene and is therefore expressed in place of the target gene. 
a)

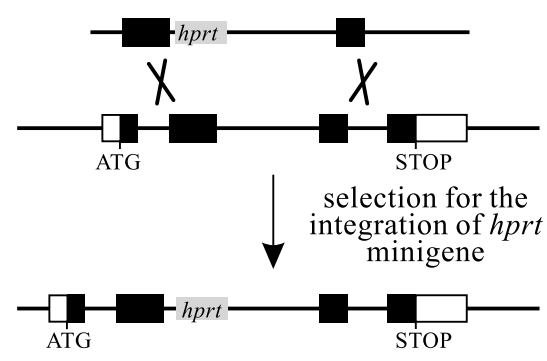

b)

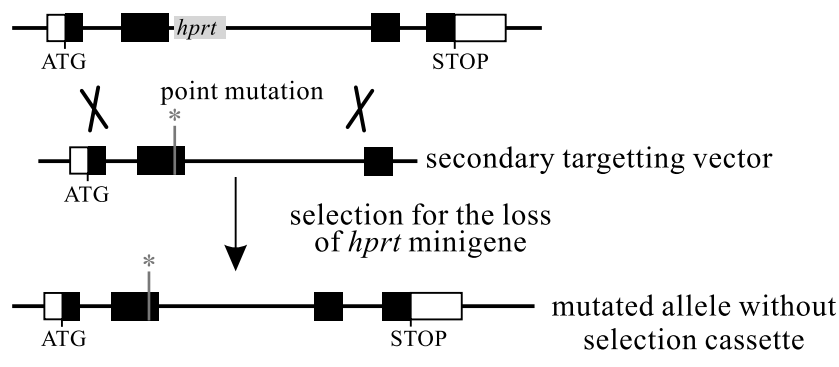

Double replacement

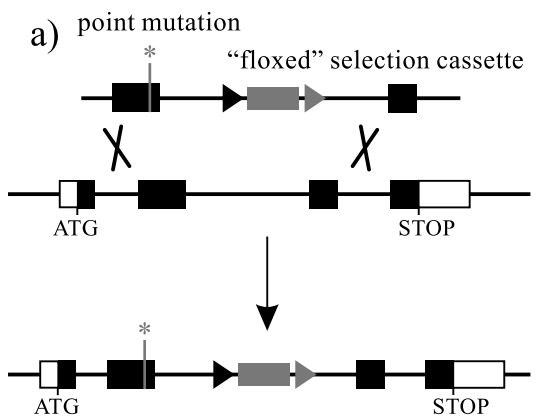

b)

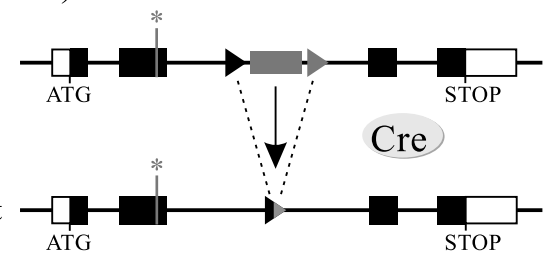

Cre/loxP system

Fig. 3 - "Clean" mutations. The persistence in a modified allele of a selection cassette with its own promoter and regulatory sequences may affect the target locus and surrounding loci. Creation of subtle mutations (point mutations, small deletions and insertions, ...) therefore requires elimination of the selection cassette. The two strategies most used to create this type of modifications are presented in this figure. Left panels: the double replacement strategy. This approach requires the use of $h \mathrm{prt}^{-}$ES cell lines. The first step (a) consists in introducing a cassette expressing the $h p r t$ gene in the target gene. The recombinant cells $\left(\mathrm{hprt}^{+}\right)$are selected in the presence of HAT. In the second step (b), these cells are transfected with a replacement vector presenting a subtle mutation and devoid of a selection cassette. The homologous recombination event results in the loss of the hprt expression cassette, an event selected in the presence of 6-TG. The use of other replacement vectors carrying different modifications permits the rapid creation of several alleles for the same target gene. Right panels: use of the Cre/loxP system (see Fig. 4). In the first step (a), the target gene is modified by a target vector with a subtle mutation and a "floxed" selection cassette, i.e. surrounded by 2 lox $P$ sites in the same orientation. Then (b) the transient expression of Cre recombinase in the recombinant cells induces deletion of the selection cassette. Apart from the desired subtle modification, only one loxP site of $34 \mathrm{pb}$ persists in the final modified allele. The position of this loxP site is chosen so that it does not interfere with the expression of the target gene (generally in an intron). 


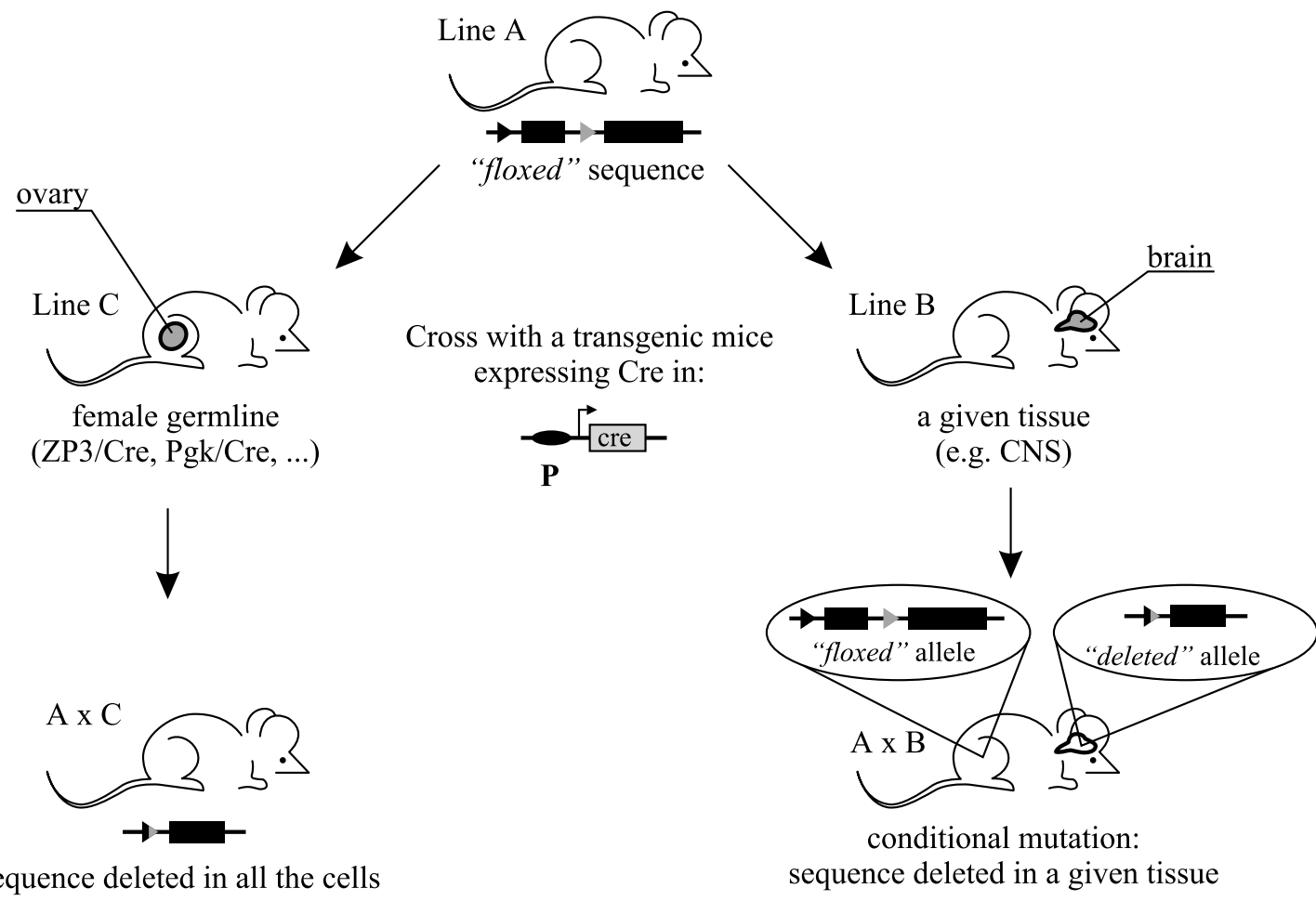

Fig. 5 - The Cre/loxP system in vivo. The Cre/loxP system can be used directly in the mouse. The most common applications of this approach are illustrated in this figure and require the use of two types of mouse. The first mouse (line A), obtained after homologous recombination in the ES cells, carries in its genome a "floxed"' sequence (exons, regulatory sequences, promoter, selection cassette,...). The other is a transgenic mouse expressing the Cre recombination under the control of a given promoter. If this promoter directs the expression of Cre in the female germ line (line C), the animals issued from a cross between a female $\mathrm{C}$ and a male $\mathrm{A}$ will carry the deletion in all their cells, the recombination event having taken place at the zygote stage through the recombinase accumulated in the oocyte. If the promoter directs the expression of Cre in one or several cell types (line B), the animals born from a cross between a mouse A and a mouse $\mathrm{C}$ will be mosaics: the cell types expressing the recombinase will carry a deleted allele whereas the other cells of the animal will carry a "floxed" allele. CNS: central nervous system; P: promoter. 